\title{
GMR
}

\section{Genetic association of sequence variation in exon 3 of the swine leukocyte antigen- $D Q A$ gene with piglet diarrhea in Large White, Landrace, and Duroc piglets}

\author{
Q.L. Yang ${ }^{1}$, X.Y. Huang ${ }^{1}$, J.J. Kong ${ }^{2}$, S.G. Zhao ${ }^{1}$, L.X. Liu ${ }^{3}$ and S.B. Gun ${ }^{1,4}$ \\ ${ }^{1}$ College of Animal Science and Technology, Gansu Agricultural University, \\ Lanzhou, China \\ ${ }^{2}$ Yongjing Country Agriculture and Animal Husbandry Bureau, Linxia, China \\ ${ }^{3}$ College of Life Science and Engineering, Northwest University for Nationalities, \\ Lanzhou, China \\ ${ }^{4}$ Gansu Research Center for Swine Production Engineering and Technology, \\ Lanzhou, China \\ Corresponding author: S.B. Gun \\ E-mail: gunsbao056@126.com
}

Genet. Mol. Res. 15 (3): gmr. 15038673

Received March 30, 2016

Accepted May 5, 2016

Published August 18, 2016

DOI http://dx.doi.org/10.4238/gmr.15038673

Copyright (C) 2016 The Authors. This is an open-access article distributed under the terms of the Creative Commons Attribution ShareAlike (CC BY-SA) 4.0 License.

\begin{abstract}
Piglet diarrhea is one of the primary factors that affects the benefits of the swine industry. Recent studies have shown that exon 2 of the swine leukocyte antigen- $D Q A$ gene is associated with piglet resistance to diarrhea; however, the contributions of additional exon coding regions of this gene remain unclear. Here, we detected and sequenced variants in the exon 3 region and examined their associations with diarrhea infection in 425 suckling piglets using the polymerase chain reaction-single-strand conformational polymorphism
\end{abstract}


and sequencing analysis. The results revealed that exon 3 of the swine leukocyte antigen- $D Q A$ gene is highly polymorphic and pivotal to both diarrhea susceptibility and resistance in piglets. We identified 14 genotypes (AA, AB, BB, BC, CC, EE, EF, BE, BF, CF, DD, DH, $\mathrm{GG}$, and $\mathrm{GF}$ ) and eight alleles $(\mathrm{A}-\mathrm{H})$ that were generated by 14 nucleotide variants, eight of which were novel, and three nucleotide deletions. Statistical analyses revealed that the genotypes $\mathrm{AB}$ and $\mathrm{EF}$ were associated with resistance to diarrheal disease $(P<0.05)$, and the genotype DD may contribute to diarrhea susceptibility but was unique to Large White pigs $(\mathrm{P}>0.05)$. These results elucidate the genetic and immunological background to piglet diarrhea, and provide useful information for resistance breeding programs.

Key words: $S L A-D Q A$; Variation; Genetic diversity; Resistance; Piglet diarrhea

\section{INTRODUCTION}

Piglet diarrhea is one of the primary factors that affects the benefits of the swine industry (Morris et al., 2002), and both genetic predisposition and environmental pathogens contribute to its development. Measures including strengthening feed management, medication, and vaccine prevention can prevent incidences of diarrhea to some extent, but increasing drug and vaccine resistance has seriously hampered efforts to prevent piglet diarrhea, and increased the feeding management requirements for piglets (Abu-Raddad et al., 2009). Therefore, genetically improving the diarrhea resistance of piglets is important.

Research has shown that some immune response gene families are strongly associated with diarrhea in neonatal and postweaning piglets (Peng et al., 2007; Jacobsen et al., 2010; Bao et al., 2012; Liu et al., 2013; Zhao et al., 2014). Swine leukocyte antigen (SLA) is a member of an immune response-related multigene family, spans the centromere of swine chromosome 7, and consists of class I, II, and III genes that participate in combating the invasion of pathogens and infectious diseases (Renard et al., 2006). The roles of SLA-encoded genes in the development and control of immunological diseases are well established, particularly those of SLA II genes (Lunney et al., 2009; Shinkai et al., 2012; Ye et al., 2012; Liu et al., 2015a). Previous studies on the molecular characterization of SLA II genes have mainly focused on the functional region of exon 2, as amino acid substitutions here are known to play crucial roles in the formation of functional conformations and in processing foreign peptides (Luetkemeier et al., 2009).

$S L A-D Q A$ is a SLA class II gene that is expressed at the mRNA and protein levels, is approximately $5.5 \mathrm{~kb}$ in length, and encodes a polypeptide of 255 amino acid residues (Ho, 2006). This gene contains four exons and three introns: exon 1 encodes a signal peptide, exons 2 and 3 encode corresponding external cellular $\alpha 1$ and $\alpha 2$ domains, and exon 4 encodes both the transmembrane and cytoplasmic domains. Although recent study has increased our knowledge of the association between $D Q A$ exon 2 genetic variation and piglet diarrhea (Yang et al., 2013; Huang et al., 2015; Liu et al., 2015b), it is widely accepted that polymorphic loci do not act alone in determining disease resistance, but rather as part of an intrinsic network (Stumpf, 2004; Bahlo et al., 2006). As both SLA-DRA and SLA-DRA-DQA haplotypes are linked to diarrhea resistance in Large White, Landrace, Duroc, and Yantai Black piglets (Yang

Genetics and Molecular Research 15 (3): gmr.15038673 
et al., 2014, 2015), it is probable that variants in additional $S L A-D Q A$ exon coding regions act as a linked intrinsic network to contribute to diarrhea disease.

Comparisons with SLA-DQA full-length cDNA sequences in the IPD-SLA (https:// www.ebi.ac.uk/ipd/mhc/sla/) and GenBank databases have revealed that, interestingly, 24 single nucleotide polymorphic sites have been identified in exon 3 of $S L A-D Q A$, in which the external cellular $\alpha 2$ domain and $\beta 2$ domains intertwine and form intra-chain disulfide bonds to maintain $D Q A$ molecular structure. However, considerable sequencing effort has been focused on miniature pigs and local breeds including Yucatan, NIH, Sinclair, Meishan, Taihu, the Bama miniature pig, Korean native pigs, and Asian wild boar, and only a handful of sequences have been obtained from Large White and Landrace pigs (Ho et al., 2010).

Large White, Landrace, and Duroc pigs are bred intensively in large-scale breeding systems. Therefore, given the risks inherent in large-scale intensive breeding and the crucial role of SLA-DQA additional exon coding regions in diarrheal disease, here we characterized variations in the exon 3 region using the polymerase chain reaction-single-strand conformational polymorphism (PCR-SSCP) method, and examined their associations with piglet diarrhea. This will elucidate the genetic and immunological background to piglet diarrhea, and provide useful information for resistance breeding programs.

\section{MATERIAL AND METHODS}

\section{Samples and data collection}

All of the animal procedures were conducted in accordance with the Guide for Chinese Feeding Standard of Swine and approved by the Ministry of Agriculture Feed Industry Center, China Agricultural University (Beijing, China). Ear tissue samples were collected from three introduced commercial pig strains (Large White, $\mathrm{N}=148$; Landrace, $\mathrm{N}=146$; Duroc, $\mathrm{N}=$ 131), as in our earlier study (Yang et al., 2013). The piglets used were the June 2010 progeny of 17 Large White, 17 Landrace, and 14 Duroc sows that were maintained under similar forage, feed management, and disease control systems, and were used to investigate the association between exon 2 of SLA-DQA and diarrhea.

Fecal traits were recorded for all of the piglets over the entire 28-day suckling period. Symptoms of diarrhea were determined by visually scoring the consistency of feces daily on a scale of 0-3 based on Kelly et al. (1990) (0, normal, solid feces; 1, slight diarrhea, soft and loose feces; 2 , moderate diarrhea, semi-liquid feces; 3 , severe diarrhea, liquid and unformed feces). The highest score attained by each piglet during the observation period was taken from these observations.

\section{Primers and PCR amplification}

Genomic DNA was extracted using a standard phenol-chloroform methodology (Sambrook and Russell, 2001) and concentrations were determined using a NanoDrop ${ }^{\circledR}$ ND-1000 spectrophotometer (NanoDrop Technologies, Inc. Wilmington, DE, USA). A PCR was performed to amplify a 388-bp fragment of $S L A-D Q A$ that included a 68 -bp fragment of intron 2, a 282-bp fragment of exon 3, and a 38-bp fragment of intron 3 using the primers $S L A-D Q A e 3 F$ (5'-CAGGACACAACGCAGAACTCA-3') and SLA-DQAe3R (5'-GAAACAAAGGTTCCAAAAGGG-3'). The primers were designed according to the published SLA-DQA sequence in GenBank (accession No. AY303988).

Genetics and Molecular Research 15 (3): gmr.15038673 
We performed the PCR in a $25-\mu \mathrm{L}$ reaction volume that contained $10 \mathrm{pM}$ each primer, $2.5 \mathrm{mM}$ dNTPs, 5 U Taq DNA polymerase (TaKaRa, Dalian, China), 10X reaction buffer (containing $15 \mathrm{mM} \mathrm{MgCl}$ ), and 50-100 $\mathrm{ng} / \mu \mathrm{L}$ genomic DNA. PCR amplifications were conducted in the following sequence: one pre-denaturation cycle at $94^{\circ} \mathrm{C}$ for $2 \mathrm{~min} ; 30$ cycles of denaturation at $94^{\circ} \mathrm{C}$ for $30 \mathrm{~s}$; annealing at $56.8^{\circ} \mathrm{C}$ for $30 \mathrm{~s}$; elongation at $72^{\circ} \mathrm{C}$ for $30 \mathrm{~s}$, and one post-elongation cycle at $72^{\circ} \mathrm{C}$ for $10 \mathrm{~min}$ in a Biometra ${ }^{\circledR}$ TGradient PCR Thermocycler (Analytik Jena AG, Jena, Germany). The PCR amplicon segments were resolved by $2 \%$ agarose gel electrophoresis (Biowest Agarose, Spain).

\section{Genotyping by SSCP, cloning, and sequencing}

Genotypes of the amplified exons were analyzed using the SSCP procedure (Sambrook and Russell, 2001). Approximately $2 \mu \mathrm{L}$ PCR product was mixed with $6 \mu \mathrm{L}$ denaturing solution [ $98 \%$ formamide, $0.025 \%$ bromophenol blue, $0.025 \%$ xylene cyanol, and $10 \mathrm{mM}$ ethylenediaminetetraacetic acid (EDTA)], denatured for $10 \mathrm{~min}$ at $98^{\circ} \mathrm{C}$, and then immediately chilled on ice for $10 \mathrm{~min}$. Denatured DNA samples were run on polyacrylamide gels $(17 \mathrm{x}$ $17 \mathrm{~cm}$ ) in a $1 \mathrm{X}$ Tris-borate/EDTA buffer in optimal conditions, and were then silver-stained following Byun et al. (2009).

To detect nucleotide variations in $S L A-D Q A$, PCR artifacts from homozygous individuals were directly sequenced. Putative heterozygotes were purified using a DNA Fragment Quick Recovery Kit (TaKaRa), ligated into pMD $^{\circledR} 19-\mathrm{T}$ vectors $(\mathrm{TaKaRa})$, and transformed into Escherichia coli DH5 $\alpha$ strains according to the manufacturer instructions. For clone selection, the transformed bacteria were plated onto Luria-Bertani medium containing $100 \mathrm{mg} / \mathrm{mL}$ ampicillin, $20 \mathrm{mg} / \mathrm{mL}$ X-gal solution, and $200 \mathrm{mg} / \mathrm{mL}$ isopropyl $\beta$-D-1-thiogalactopyranoside. Initially, at least 10 positive colonies were picked from each transformation and incubated in a vibrating rotary incubator $(200 \mathrm{rpm})$ overnight at $37^{\circ} \mathrm{C}$ in a $4-\mathrm{mL}$ Luria-Bertani broth containing $100 \mathrm{mg} / \mathrm{mL}$ ampicillin. The targeted insertions and sequences of both alleles were verified using the PCR-SSCP approach as described by Zhou and Hickford (2008). Insertions with a SSCP pattern that matched genomic DNA typing (two clones for each genotype) were selected for subsequent sequencing by Sangon Biotech Co. Ltd. (Shanghai, China).

\section{Statistical analysis}

Sequence alignment and translation were performed using the software MEGA 5.0 (Tamura et al., 2011). Allele frequencies, effective allele numbers, the observed and expected heterozygosity, and potential deviations from the Hardy-Weinberg equilibrium were calculated using POPGENE version 1.32 (Yeh et al., 1997). The polymorphism information content (PIC) was calculated following Botstein et al. (1980).

Relative rates of synonymous $\left(\mathrm{d}_{\mathrm{s}}\right)$ and nonsynonymous $\left(\mathrm{d}_{\mathrm{N}}\right)$ substitutions were estimated using the method of Nei and Gojobori (1986) with a Jukes-Cantor correction. We performed Z-tests for selection between $\mathrm{d}_{\mathrm{S}}$ and $\mathrm{d}_{\mathrm{N}}$ (Nei and Kumar, 2000), and pairwise nucleotide and amino acid distances were estimated using the Kimura-2-parameter and Poisson models in MEGA 5.0.

Associations between genotypes and the prevalence of piglet diarrhea were determined using a least squares method implemented as part of a general linear model in SAS version 8.01 (SAS Institute Inc., Cary, NC, USA). The statistical model used was as follows:

Genetics and Molecular Research 15 (3): gmr.15038673 


$$
Y_{i j k l}=\mu+B_{i}+S_{j}+L_{k}+G_{1}+e_{i j k l}
$$

(Equation 1)

where $Y_{i j k l}$ is the highest observed diarrhea score, $\mu$ is the overall breed mean, $B_{i}$ is the fixed effect of breed, $S_{j}$ is the fixed effect of gender, $L_{k}$ is the fixed effect of litter (sow), $G_{l}$ is the genetic effect of genotype, and $e$ is the random residual effect.

\section{RESULTS}

\section{Polymorphisms in exon 3 of $S L A-D Q A$}

We identified 14 SSCP patterns (AA, AB, BB, BC, CC, EE, EF, BE, BF, CF, DD, DH, $\mathrm{GG}$, and GF) for exon 3 that were confirmed as eight alleles (A-H) (Figure 1). There were 13, 11, and 9 genotypes in Large White, Landrace, and Duroc pigs, respectively, and AA and BB were the most frequent, being present in all three breeds. The DD and DH genotypes were only present in eight and four Large White pigs, respectively (Figure 2A). The B allele was dominant in all of the breeds analyzed, while $\mathrm{D}$ and $\mathrm{H}$ were only present in Large White pigs (Figure 2B).

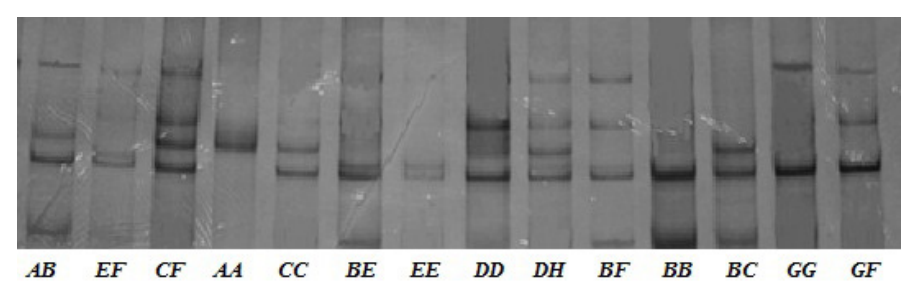

Figure 1. PCR-SSCP analysis of PCR amplified products for exon 3 of the $S L A-D Q A$ gene.
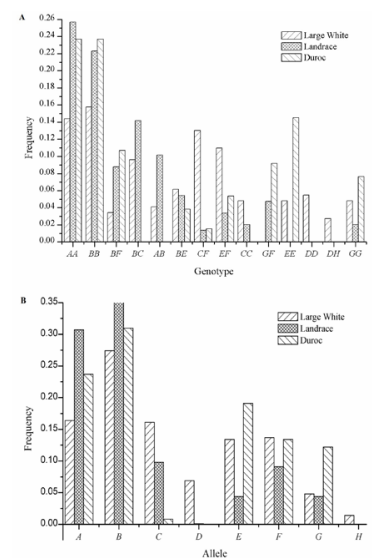

Figure 2. Distribution for genotype and allele frequencies in exon 3 of the $S L A-D Q A$ gene in the three pig breeds.

\section{Sequence variations in exon 3 of $S L A-D Q A$}

Seventeen of 282-bp nucleotide variants were identified in the exon 3 region in the three breeds (Table 1). By deducing the amino acid alignments of these alleles, we found variation in eight amino acids and two deletions (Figure 3), with $6.03 \%$ variability at the nucleotide level and $10.64 \%$ variability at the amino acid level. 


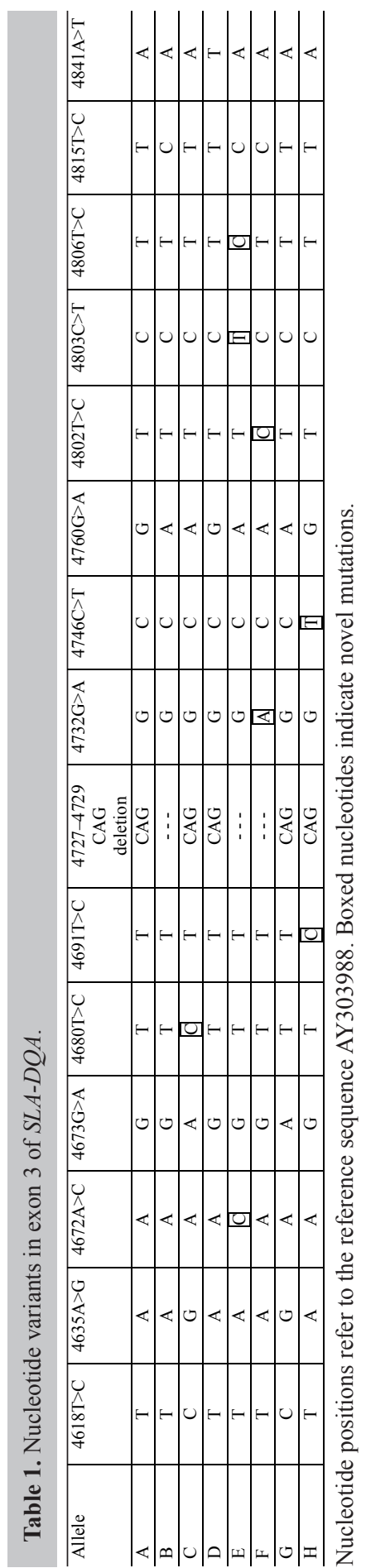

Genetics and Molecular Research 15 (3): gmr.15038673 


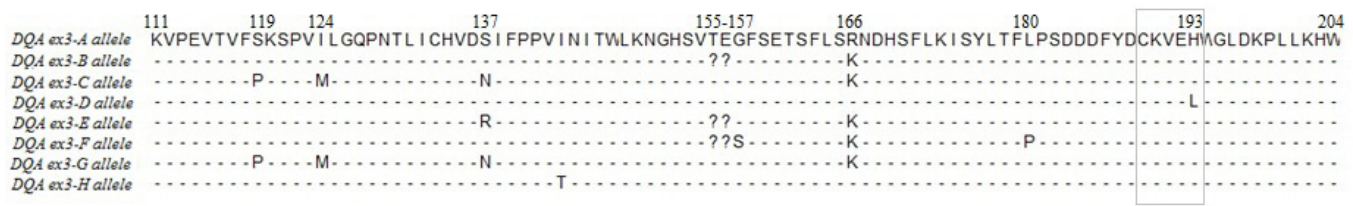

Figure 3. Amino acid sequences in exon 3 of the $S L A-D Q A$ alleles identified from the three pig breeds. Question mark indicate amino acid deletion. Gray box denote the putative stalk segment of transmembrane domains combine with $\alpha 2$ domain.

After comparing 67 SLA-DQA full-length cDNA sequences (34 from IPD-SLA and 33 from GenBank), eight variants (c.4672A $>C$, c.4803C $>$ T, and c.4806T $>C$ in the E allele; c. $4680 \mathrm{~T}>\mathrm{C}$ in the $\mathrm{C}$ allele; c.4732G $>\mathrm{A}$ and c. $4802 \mathrm{~T}>\mathrm{C}$ in the $\mathrm{F}$ allele; and c.4691T $>\mathrm{C}$ and c. $4746 \mathrm{C}>\mathrm{T}$ in the $\mathrm{H}$ allele) were found to be novel (Table 1). The allele sequences were deposited in GenBank with the following accession Nos.: KT005556, KT005567, JX840407, KT005569, JX840408, JX840409, KT005568, and JX840410.

We estimated allele diversity at the SLA-DQA loci for the three breeds (Table 2), and found that the number of effective alleles ranged from 3.46 to 5.82 , which is lower than that previously observed for these breeds. The observed heterozygosity was lower than the expected heterozygosity and ranged from 0.31 to 0.50 , and a high PIC value $(>0.67)$ was also found.

Table 2. Genetic characteristics of exon 3 in $S L A-D Q A$ in three pig breeds.

\begin{tabular}{l|c|c|c}
\hline Breed & Large White & Landrace & Duroc \\
\hline $\mathrm{N}$ & 146 & 148 & 131 \\
\hline$N_{\mathrm{E} / N_{\mathrm{A}}}$ & $5.82 / 6$ & $3.46 / 6$ & $4.53 / 6$ \\
\hline$H_{\mathrm{O} / H_{\mathrm{E}}}$ & $0.50 / 0.83$ & $0.48 / 0.71$ & $0.31 / 0.78$ \\
\hline $\mathrm{PIC}$ & 0.81 & 0.67 & 0.75 \\
\hline$\chi^{2}$ & $493^{* *}$ & $172^{* *}$ & $284^{* *}$ \\
\hline
\end{tabular}

$N_{\mathrm{E}}$, effective allele number; $N_{\mathrm{A}}$, observed number of alleles; $H_{\mathrm{O}}$, observed heterozygosity; $H_{\mathrm{E}}$, expected heterozygosity; PIC, polymorphism information content; $\chi^{2}$, chi-square test for the Hardy-Weinberg equilibrium. **Genetic disequilibrium, $\mathrm{P}<0.01$.

The mean nucleotide and amino acid distances between the allele sequences were 0.016 and 0.042 , respectively, and the nucleotide and amino acid distances for pairwise sequence comparisons ranged from 0.004 to 0.029 and 0.011 to 0.071 , respectively (Table 3 ). We observed low ratios of synonymous and nonsynonymous substitutions $\left(\mathrm{d}_{\mathrm{N}} / \mathrm{d}_{\mathrm{S}}<1\right)$ among the $S L A-D Q A$ alleles, which were not significantly different (Table 3).

Table 3. Synonymous and nonsynonymous substitutions in exon 3 of $S L A-D Q A$ alleles.

\begin{tabular}{l|c|c|c|c|c}
\hline $\mathrm{N}$ & $\mathrm{d}_{\mathrm{N}} \pm$ SEM & $\mathrm{ds} \pm$ SEM & $\mathrm{d}_{\mathrm{N}} / \mathrm{ds}$ & Nucleotide $(\mathrm{D} \pm \mathrm{SEM})$ & Amino acid $(\mathrm{D} \pm \mathrm{SEM})$ \\
\hline 94 & $0.014 \pm 0.001(0.000-0.023)$ & $0.025 \pm 0.003(0.000-0.067)$ & $0.566^{\mathrm{ns}}$ & $0.016 \pm 0.001(0.004-0.029)$ & $0.042 \pm 0.003(0.011-0.071)$ \\
\hline
\end{tabular}

Average distances of $\mathrm{d}_{\mathrm{S}}$ and $\mathrm{d}_{\mathrm{N}}$ and their ratios were calculated using the Nei-Gojobori method with a Jukes-Cantor correction. Significance of an excess in $\mathrm{d}_{\mathrm{N}}$ or $\mathrm{d}_{\mathrm{S}}$ was tested by the $Z$-test. Pairwise rates are shown in parentheses. ns, not significant; N, number of codons; D, distances for pairwise sequence comparisons.

\section{$S L A-D Q A$ genotypes and piglet diarrheal disease}

Our statistical model revealed that breed, sex, and litter (sow) did not affect piglet diarrheal infection probability $(\mathrm{P}>0.05)$. However, a significant correlation between genotype 
and diarrheal infection was observed $(\mathrm{P}<0.01)$. Means and standard errors of least squares for diarrheal score for the different genotypes are shown in Table 4; scores for $\mathrm{AB}(0.38 \pm 0.20)$ and $\mathrm{EF}(0.47 \pm 0.20)$ were significantly lower than those for $\mathrm{BB}(0.97 \pm 0.10), \mathrm{BC}(1.18 \pm$ $0.15), \mathrm{BE}(1.14 \pm 0.21), \mathrm{EE}(1.16 \pm 0.19), \mathrm{DD}(1.73 \pm 0.30)$, and $\mathrm{GG}(1.29 \pm 0.37)(\mathrm{P}<0.01)$, and were also lower than those for AA $(0.89 \pm 0.09)(\mathrm{P}<0.05)$, whereas the diarrheal score for DD was significantly higher than for the other genotypes $(1.73 \pm 0.30)$. Genotype DH was excluded from the linear contrast analysis because of the small sample size $(\mathrm{N}<5)$.

Table 4. Least square means and standard errors of piglet diarrhea scores in different genotypes of SLA-DQA.

\begin{tabular}{l|c|c}
\hline Genotype & Number & Diarrhea score \\
\hline AA & 89 & $0.89 \pm 0.09^{\mathrm{b}}$ \\
\hline BB & 87 & $0.97 \pm 0.10^{\mathrm{B}}$ \\
\hline AB & 21 & $0.38 \pm 0.20^{\mathrm{Aa}}$ \\
\hline BC & 35 & $1.18 \pm 0.15^{\mathrm{Bb}}$ \\
\hline CC & 10 & $0.54 \pm 0.34^{\mathrm{ab}}$ \\
\hline BE & 22 & $1.14 \pm 0.21^{\mathrm{Bb}}$ \\
\hline CF & 23 & $0.73 \pm 0.41^{\mathrm{ab}}$ \\
\hline EF & 28 & $0.47 \pm 0.20^{\mathrm{Aa}}$ \\
\hline DD & 8 & $1.73 \pm 0.30^{\mathrm{Bb}}$ \\
\hline EE & 19 & $0.86 \pm 0.20^{\mathrm{ab}}$ \\
\hline BF & 26 & $1.16 \pm 0.19^{\mathrm{Bb}}$ \\
\hline DH & 32 & $0.63 \pm 0.17^{\mathrm{ab}}$ \\
\hline GG & 4 & $2.33 \pm 0.48$ \\
\hline
\end{tabular}

$\mathrm{A}$ and $\mathrm{B}$ in the same row indicates a significant difference at $\mathrm{P}<0.01$ among different genotypes; $\mathrm{a}$ and $\mathrm{b}$ in the same row indicates a significant difference at $\mathrm{P}<0.05$ among different genotypes.

\section{DISCUSSION}

In this study, we characterized the diversity of $S L A-D Q A$ by detecting and sequencing exon 3 using PCR-SSCP, and obtained 14 SSCP patterns and eight alleles. Significantly, the effective allele values were lower than those previously observed, perhaps because of unevenly distributed genotype frequencies; these values are, however, consistent with the Hardy-Weinberg equilibrium based on chi-square tests. The observed heterozygosity was also lower than expected, which suggests that many homozygous individuals occur in Large White, Landrace, and Duroc pigs, and that factors such as selection, mutation, and genetic drift may have little effect on these breeds. These results are in contrast with those obtained from a previous investigation of $S L A-D Q A$, which reported high heterozygosity in Landrace (0.95) and Yorkshire (0.80) pigs (Le et al., 2012).

We found 17 polymorphic nucleotide sites in exon 3 in our Large White, Landrace, and Duroc pigs. The comparisons with SLA-DQA full-length cDNA sequences in the IPD-SLA and GenBank databases showed that, to date, only 24 single nucleotide polymorphic sites have been identified in this region, so exon 3 is probably a hypervariable region in these pig breeds. In addition, a high PIC value ( $>0.67)$ was observed at this locus in the three breeds, which provides further evidence that exon 3 is highly polymorphic. Of the 17 nucleotide variants found in exon 3, eight (c.4672A $>$ C, c. $4680 \mathrm{~T}>\mathrm{C}$, c. $4691 \mathrm{~T}>\mathrm{C}, \mathrm{c} .4732 \mathrm{G}>\mathrm{A}, \mathrm{c} .4746 \mathrm{C}>\mathrm{T}, \mathrm{c} .4802 \mathrm{~T}>\mathrm{C}$, c. $4803 \mathrm{C}>\mathrm{T}$, and c.4806T $>\mathrm{C}$ ) were novel, which was a greater number of $D Q A$ alleles than was found in exon 2 in a previous study (Yang et al., 2013). Liu et al. (Liu LX, Gun SB, Zhao SG, Lu HN, et al., unpublished results) identified four nucleotide variants (c.4618T $>$ C,

Genetics and Molecular Research 15 (3): gmr.15038673 
c. $4635 \mathrm{~A}>\mathrm{G}, \mathrm{c} .4673 \mathrm{G}>\mathrm{A}$, and c.4760G $>\mathrm{A}$ ) and three nucleotide deletions (c.4727-4729 CAG) that formed only three alleles in 471 Bamei, Juema, and Gansu Black pigs (GenBank accession Nos. KP324797-KP324799), while Huang et al. (2015) identified seven polymorphic sites identical to those found by Liu et al. (Liu LX, Gun SB, Zhao SG, Lu HN, et al., unpublished results) and one additional novel mutation (c.4617T $>$ C) that formed six alleles in 290 Yantai Black pigs (GenBank accession Nos. KM485555-KM485560). According to these results, the novel variants identified in the present study appear to be specific to Landrace, Large White, and Duroc pigs.

Exon 3 of $S L A-D Q A$ encodes the external cellular $\alpha 2$ domain, in which the $\alpha 2$ and $\beta 2$ domains intertwine and form intra-chain disulfide bonds to maintain $D Q A$ molecular structure (Ho, 2006). In the present study, we confirmed that a statistical association exists between the genotypes of exon 3 and diarrhea susceptibility and resistance. Genotype DH had the highest score, but was excluded from the disease-association analysis because it had a very small sample size $(\mathrm{N}<5)$. We found that the heterozygous genotypes $\mathrm{AB}$ and $\mathrm{EF}$ had a high probability of diarrhea resistance, and consequently will provide a selective advantage in Large White, Landrace, and Duroc breeds. This result is supported by the fact that individuals that are heterozygous at major histocompatibility complex loci have higher adaptive immunity against infectious diseases than homozygous individuals (McClelland et al., 2003; Spurgin and Richardson, 2010; Trowsdale, 2011). Heterozygosity of BoLA-DQA1 is associated with resistance to proviral bovine leukemia in Japanese Black cattle (Miyasaka et al., 2013) and mastitis in dairy cattle (Takeshima et al., 2008). Curiously, however, homozygous genotypes such as CC in exon 2 of $D Q A$ in Large White, Landrace, and Duroc pigs (Yang et al., 2013), DQA*03xa0303xa03 in exon 2 of $D Q A$ in Bamei, Juema, and Gansu Black pigs (Liu et al., 2015b), and BB in exon 2 of $D Q A$ in Yantai Black pigs (Yang et al., 2015) are associated with low diarrheal scores, and are probably resistant genotypes. These results confirm the fact that resistant homozygotes play important roles in pathogen defense.

Our results suggest that DD genotype may confer a high susceptibility to diarrhea only in Large White pigs, as their scores were higher than those of the other breeds. SLA II genes share four sequences [PEVTV or PTVTV(I), NT(I)LIC or NLLVC, WLK(R)NG or WFRNG, and $\mathrm{CK}(\mathrm{Q}) \mathrm{VEH}$ or $\mathrm{CRVEH}]$ that consist of five amino acids in the $\alpha 2$ and $\beta 2$ domains, which are probably transitional zones for $\alpha$-helix or $\beta$-folding (Tan, unpublished results). Interestingly, we found that mutation c. $4841 \mathrm{~A}>\mathrm{T}$ in the $\mathrm{D}$ allele was located in the transitional CKVEH zone (marked with a gray box in Figure 3), and generates an amino acid substitution of histidine to leucine (p.193His> Leu), which is thought to be functionally related to proteincoding stability (Gu et al., 2015). This may explain why genotypes containing this allele were susceptible to diarrhea. Therefore, resistant genotypes may be shared between Large White, Landrace, and Duroc breeds, while susceptible genotypes differ between breeds. Fortunately, only eight Large White individuals with these susceptible genotypes were identified in our study. Diarrhea susceptibility in Yantai Black pigs has been attributed to the AD genotype in SLA-DQA (Huang et al., 2015); however, the mutations reported in alleles A and D of $D Q A$ exon 3 are inconsistent with the results of the present study. There are two possible explanations for this, which are not mutually exclusive: firstly, the three widely available commercial pig breeds used here differ from the indigenous Yantai Black pig because of artificial selection; secondly, nucleotide mutations can alter mRNA expression and function, which affects normal protein function (Gu et al., 2015). Therefore, the results of future studies should be interpreted cautiously, until the expression and mechanisms of SLA-DQA-related diarrhea resistance are elucidated.

Genetics and Molecular Research 15 (3): gmr.15038673 
In conclusion, this study is the first to report eight novel nucleotide variants in exon 3 of $S L A-D Q A$, and reveals that variation in the external cellular $\alpha 2$ domain of exon 3 is pivotal to both diarrhea susceptibility and resistance in piglets. Our findings extend the current knowledge of $S L A-D Q A$, and will stimulate further biomedical research and diarrhea-resistant pig-breeding programs. Intragenic recombination studies have clear advantages over genedisease association studies when working on single-locus alleles (Liu et al., 2015a), and nucleotide sequence variations in other exon coding regions of $S L A-D Q A$ may also contribute to diarrhea in piglets, or might link to other SLA class II genes. Further work on obtaining full-length cDNA sequences of other SLA molecules and validating the linkage between these class II genes and diarrhea is required.

\section{Conflicts of interest}

The authors declare no conflict of interest.

\section{ACKNOWLEDGMENTS}

We thank the Bio-Technology Special Program of Gansu Agriculture and Animal Husbandry of China (\#GNSW-2008-04), the Important and Special Project of Gansu Department of Science and Technology of China (\#092NKDA036), and the Natural Science Foundation of China (\#31101682) for supporting this study.

\section{REFERENCES}

Abu-Raddad LJ, Sabatelli L, Achterberg JT, Sugimoto JD, et al. (2009). Epidemiological benefits of more-effective tuberculosis vaccines, drugs, and diagnostics. Proc. Natl. Acad. Sci. USA 106: 13980-13985. http://dx.doi. org/10.1073/pnas.0901720106

Bahlo M, Stankovich J, Speed TP, Rubio JP, et al. (2006). Detecting genome wide haplotype sharing using SNP or microsatellite haplotype data. Hum. Genet. 119: 38-50. http://dx.doi.org/10.1007/s00439-005-0114-9

Bao WB, Ye L, Pan ZY, Zhu J, et al. (2012). Microarray analysis of differential gene expression in sensitive and resistant pig to Escherichia coli F18. Anim. Genet. 43: 525-534.http://dx.doi.org/10.1111/j.1365-2052.2011.02287.x

Botstein D, White RL, Skolnick M and Davis RW (1980). Construction of a genetic linkage map in man using restriction fragment length polymorphisms. Am. J. Hum. Genet. 32: 314-331.

Byun SO, Fang Q, Zhou H and Hickford JG (2009). An effective method for silver-staining DNA in large numbers of polyacrylamide gels. Anal. Biochem. 385: 174-175. http://dx.doi.org/10.1016/j.ab.2008.10.024

Gu W, Gurguis CI, Zhou JJ, Zhu Y, et al. (2015). Functional and structural consequence of rare exonic single nucleotide polymorphisms: one story, two tales. Genome Biol. Evol. 7: 2929-2940. http://dx.doi.org/10.1093/gbe/evv191

Ho CS (2006). Molecular characterization of swine leucocyte antigen diversity in outbred pig populations. PhD. Dissertation. Baylor University. TX, USA. http://search.proquest.com/docview/305361189.

Ho CS, Martens GW, Amoss MS, Jr., Gomez-Raya L, et al. (2010). Swine leukocyte antigen (SLA) diversity in Sinclair and Hanford swine. Dev. Comp. Immunol. 34: 250-257.http://dx.doi.org/10.1016/j.dci.2009.09.006

Huang XY, Yang QL, Yuan JH and Gun SB (2015). Polymorphism and haplotype analyses of swine leukocyte antigen DQA exons 2, 3, 4, and their associations with piglet diarrhea in Chinese native pig. Genet. Mol. Res. 14: 1046110472. http://dx.doi.org/10.4238/2015.September.8.7

Jacobsen M, Kracht SS, Esteso G, Cirera S, et al. (2010). Refined candidate region specified by haplotype sharing for Escherichia coli F4ab/F4ac susceptibility alleles in pigs. Anim. Genet. 41: 21-25. http://dx.doi.org/10.1111/j.13652052.2009.01952.x

Kelly D, O'Brien JJ and McCracken KJ (1990). Effect of creep feeding on the incidence, duration and severity of postweaning diarrhoea in pigs. Res. Vet. Sci. 49: 223-228.

Le MT, Choi H, Choi MK, Nguyen DT, et al. (2012). Comprehensive and high-resolution typing of swine leukocyte antigen DQA from genomic DNA and determination of 25 new SLA class II haplotypes. Tissue Antigens 80: 528535.http://dx.doi.org/10.1111/tan.12017

Genetics and Molecular Research 15 (3): gmr.15038673 
Liu L, Wang J, Zhao Q, Zi C, et al. (2013). Genetic variation in exon 10 of the BPI gene is associated with Escherichia coli F18 susceptibility in Sutai piglets. Gene 523: 70-75. http://dx.doi.org/10.1016/j.gene.2013.02.051

Liu LX, Zhao SG, Lu HN, Yang QL, et al. (2015b). Association between polymorphisms of the swine $M H C-D Q A$ gene and diarrhoea in three Chinese native piglets. Int. J. Immunogenet. 42: 208-216. http://dx.doi.org/10.1111/iji.12186

Liu ZZ, Xia JH, Xin LL, Wang ZG, et al. (2015a). Swine leukocyte antigen class II genes (SLA-DRA, SLA-DRB1, SLA$D Q A, S L A-D Q B 1)$ polymorphism and genotyping in Guizhou minipigs. Genet. Mol. Res. 14: 15256-15266. http:// dx.doi.org/10.4238/2015.November.30.1

Luetkemeier ES, Malhi RS, Beever JE and Schook LB (2009). Diversification of porcine MHC class II genes: evidence for selective advantage. Immunogenetics 61: 119-129. http://dx.doi.org/10.1007/s00251-008-0348-5

Lunney JK, Ho CS, Wysocki M and Smith DM (2009). Molecular genetics of the swine major histocompatibility complex, the SLA complex. Dev. Comp. Immunol. 33: 362-374. http://dx.doi.org/10.1016/j.dci.2008.07.002

McClelland EE, Penn DJ and Potts WK (2003). Major histocompatibility complex heterozygote superiority during coinfection. Infect. Immun. 71: 2079-2086. http://dx.doi.org/10.1128/IAI.71.4.2079-2086.2003

Miyasaka T, Takeshima SN, Jimba M, Matsumoto Y, et al. (2013). Identification of bovine leukocyte antigen class II haplotypes associated with variations in bovine leukemia virus proviral load in Japanese Black cattle. Tissue Antigens 81: 72-82. http://dx.doi.org/10.1111/tan.12041

Morris RS, Davies PR and Lawton DE (2002). Evolution of diseases in the world's pig industry. 17th International Pig Veterinary Society Congress Proceedings, Ames.

Nei M and Gojobori T (1986). Simple methods for estimating the numbers of synonymous and nonsynonymous nucleotide substitutions. Mol. Biol. Evol. 3: 418-426.

Nei M and Kumar S (2000). Molecular evolution and phylogenetics. Oxford University Press, New York, 51.

Peng QL, Ren J, Yan XM, Huang X, et al. (2007). The g.243A $>$ G mutation in intron 17 of MUC4 is significantly associated with susceptibility/resistance to ETEC F4ab/ac infection in pigs. Anim. Genet. 38: 397-400. http://dx.doi. org/10.1111/j.1365-2052.2007.01608.x

Renard C, Hart E, Sehra H, Beasley H, et al. (2006). The genomic sequence and analysis of the swine major histocompatibility complex. Genomics 88: 96-110. http://dx.doi.org/10.1016/j.ygeno.2006.01.004

Sambrook J and Russell DW (2001). Molecular cloning: a laboratory manual, 3rd edn. Cold Spring Harbor Laboratory Press, New York, 17.

Shinkai H, Arakawa A, Tanaka-Matsuda M, Ide-Okumura H, et al. (2012). Genetic variability in swine leukocyte antigen class II and Toll-like receptors affects immune responses to vaccination for bacterial infections in pigs. Comp. Immunol. Microbiol. Infect. Dis. 35: 523-532. http://dx.doi.org/10.1016/j.cimid.2012.05.003

Spurgin LG and Richardson DS (2010). How pathogens drive genetic diversity: MHC, mechanisms and misunderstandings. Proc. Biol. Sci. 277: 979-988. http://dx.doi.org/10.1098/rspb.2009.2084

Stumpf MP (2004). Haplotype diversity and SNP frequency dependence in the description of genetic variation. Eur. J. Hum. Genet. 12: 469-477. http://dx.doi.org/10.1038/sj.ejhg.5201179

Takeshima S, Matsumoto Y, Chen J, Yoshida T, et al. (2008). Evidence for cattle major histocompatibility complex (BoLA) class II DQA1 gene heterozygote advantage against clinical mastitis caused by Streptococci and Escherichia species. Tissue Antigens 72: 525-531.http://dx.doi.org/10.1111/j.1399-0039.2008.01140.x

Tamura K, Peterson D, Peterson N, Stecher G, et al. (2011). MEGA5: molecular evolutionary genetics analysis using maximum likelihood, evolutionary distance, and maximum parsimony methods. Mol. Biol. Evol. 28: 2731-2739. http://dx.doi.org/10.1093/molbev/msr121

Trowsdale J (2011). The MHC, disease and selection. Immunol. Lett. 137: 1-8. http://dx.doi.org/10.1016/j.imlet.2011.01.002

Yang QL, Kong JJ, Wang DW, Zhao SG, et al. (2013). Swine leukocyte antigen- $D Q A$ gene variation and its association with piglet diarrhea in Large White, Landrace and Duroc. Asian-australas. J. Anim. Sci. 26: 1065-1071. http://dx.doi. org/10.5713/ajas.2013.13067

Yang QL, Zhao SG, Wang DW, Feng Y, et al. (2014). Association between genetic polymorphism in the swine leukocyte antigen-DRA gene and piglet diarrhea in three Chinese pig breeds. Asian-australas. J. Anim. Sci. 27: 1228-1235. http://dx.doi.org/10.5713/ajas.2013.13567

Yang QL, Huang XY, Yuan JH and Gun SB (2015). Polymorphisms in the SLA-DQA and DRA gene exon 2 and their association with piglet diarrhea in Chinese Yantai black pig. Philipp. Agric. Sci. 98: 253-261.

Ye L, Zi C, Pan ZY, Zhu J, et al. (2012). Investigation of the relationship between SLA-1 and SLA-3 gene expression and susceptibility to Escherichia coli $\mathrm{F} 18$ in post-weaning pigs. Comp. Immunol. Microbiol. Infect. Dis. 35: 23-30.http:// dx.doi.org/10.1016/j.cimid.2011.09.006

Yeh FC, Yang R-C, Boyle TBJ, Ye Z-H, et al. (1997). POPGENE, the user-friendly shareware for population genetic analysis. Molecular Biology and Biotechnology Centre, University of Alberta, Edmonton.

Zhao Q, Liu Y, Dong W, Zhu S, et al. (2014). Genetic variations of TAPl gene exon 3 affects gene expression and Escherichia coli F18 resistance in piglets. Int. J. Mol. Sci. 15: 11161-11171.http://dx.doi.org/10.3390/ijms150611161

Zhou H and Hickford JGH (2008). Clonal polymerase chain reaction-single-strand conformational polymorphism analysis: an effective approach for identifying cloned sequences. Anal. Biochem. 378: 111-112. http://dx.doi.org/10.1016/j. ab.2008.04.005

Genetics and Molecular Research 15 (3): gmr.15038673 